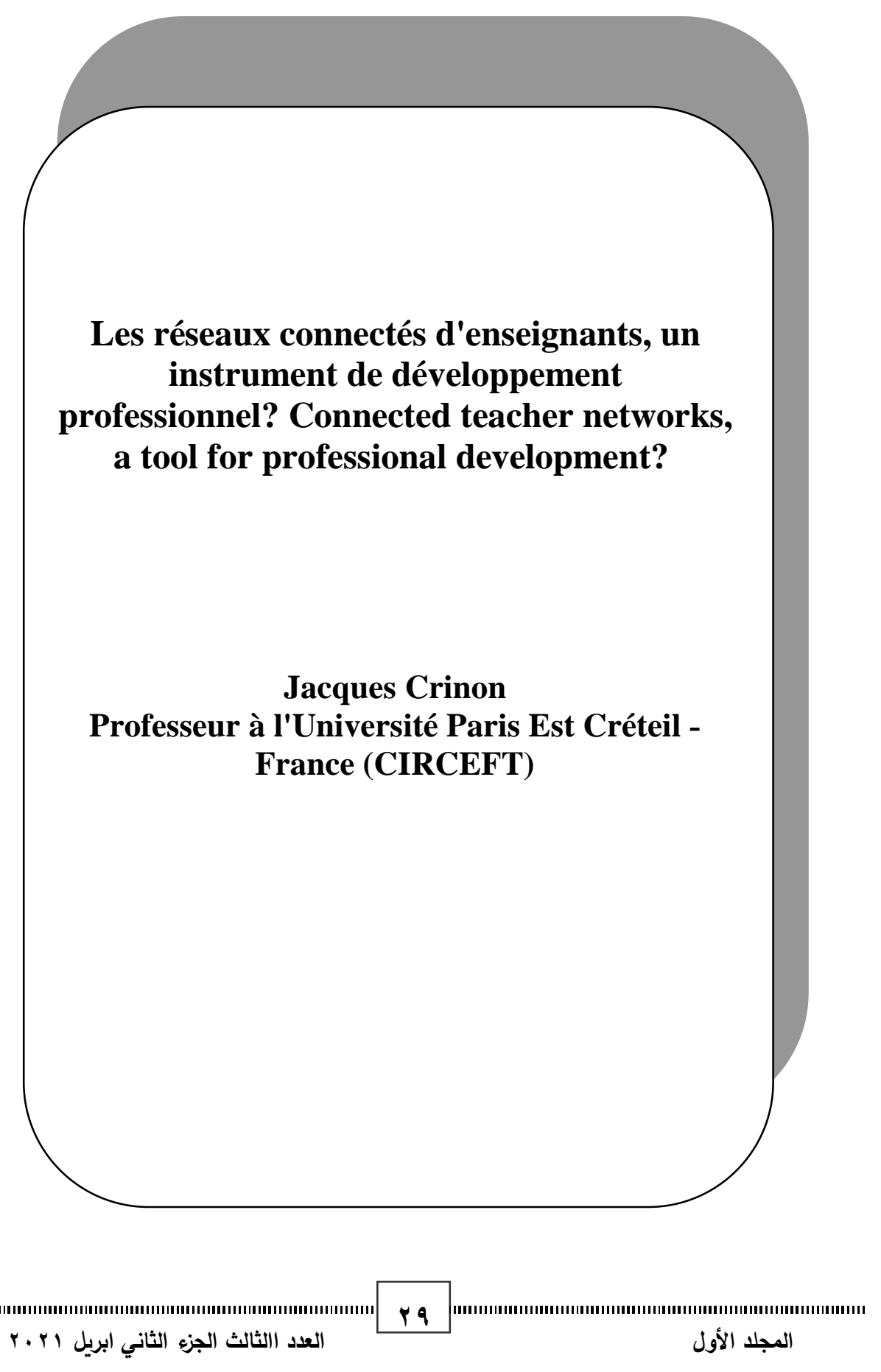


المجلة الدولية للتعليم الإكتروني

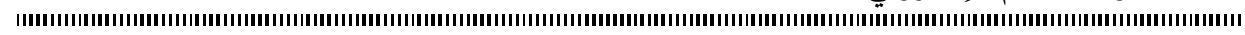

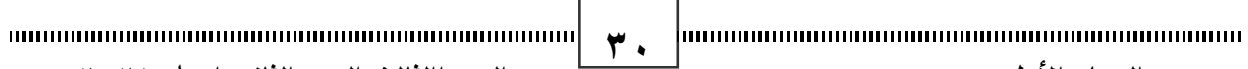

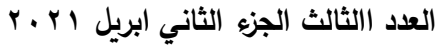
المجلد الأول 
المجلة الدولية للتعليم الإلكتروني

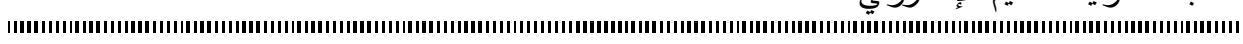

\section{Les réseaux connectés d'enseignants, un instrument de développement professionnel? Connected teacher networks, a tool for professional development?}

Jacques Crinon

\section{Résumé:}

Le développement professionnel des enseignants au long de leur carrière constitue un levier essentiel pour améliorer l'efficacité et l'équité de l'enseignement. Le travail collectif des enseignants concevant et gérant des projets et des dispositifs, en particulier à distance via des réseaux sociaux et des outils numériques de collaboration, a suscité de fortes attentes quant au développement professionnel des participants. Des effets de la participation à des réseaux d'apprentissage professionnel ou à des communautés en ligne ont-ils été mis en évidence par la recherche? Quels sont les conditions pour que des transformations se manifestent? Nous montrerons les effets souvent importants de cette participation sur la confiance en soi des participants et leur intérêt renouvelé pour l'exercice de leur métier, et des effets plus limités sur l'acquisition de savoirs et la compétence à analyser les situations d'apprentissage. Nous montrerons aussi la nécessité de considérer la participation à un réseau dans un ensemble plus large d'interactions et dans des trajets personnels de développement où celle-ci peut, à un moment, jouer un rôle décisif.

* Professeur à l'Université Paris Est Créteil, France (CIRCEFT)

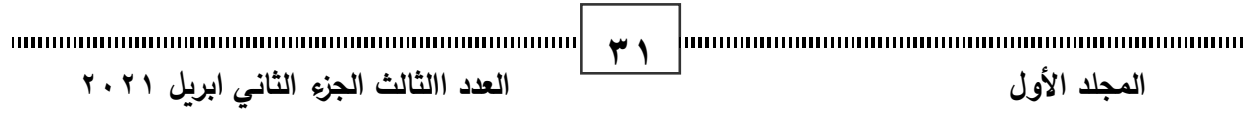




\section{Abstract:}

The professional development of teachers throughout their careers is an important lever for improving the effectiveness and equity of teaching. The collective work of teachers designing and managing projects and devices, in particular remotely via social networks and digital collaboration tools, raised high expectations regarding the professional development of participants. Have any effects of participating in professional learning networks or online communities been found in research? What are the conditions for transformations to take place? We will show the often significant effects of this participation on the participants' selfconfidence and their renewed interest in the exercise of their profession, and more limited effects on the acquisition of knowledge and the ability to analyse learning situations. We will also show the need to consider participation in a network in a larger set of interactions and in personal development paths where it can, at some point, play a decisive role.

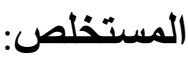

يعتبر التطوير المهني للمعلمين طوال حياتهم المهنية مهم لتحسين فعالية وعدالة

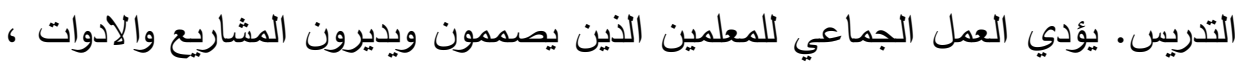

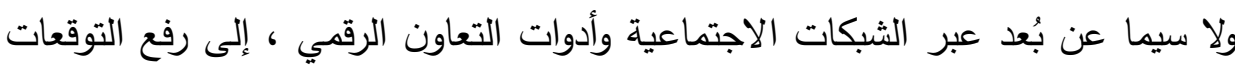

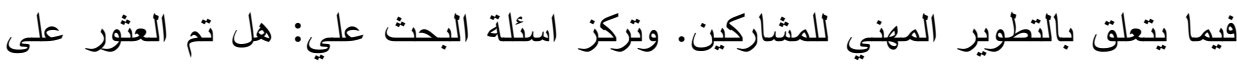
أي آثار للمشاركة في شبكات التعلم المهنية أو المجتمعات عبر الإنترنت في البحث؟ ما هي شروط حدوث التحولات؟ آنار لمنات

وقد ظهرت الآثار المهمة في كثير من الأحيان لهذه المشاركة على ثقة المشاركين

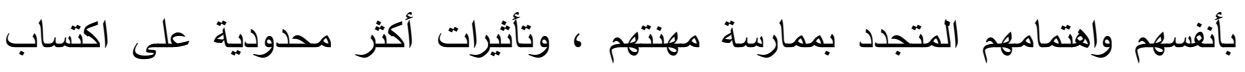
المعرفة والقدرة على تحليل مواقف التعلم. ويتضح أيضًا الحاجة إلى النظر في المشاركة في شبكة في مجموعة أكبر من التفاعلات وفي مسارات التتمية الثخصية حيث يمكنها ، في مرحلة ما ، أن تلعب دورًا حاسمًا.

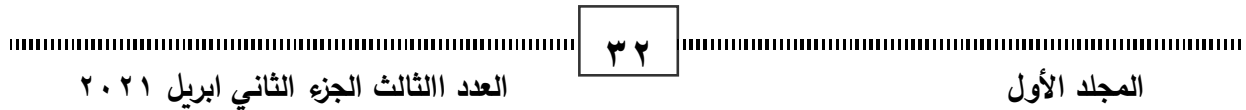




\section{Introduction}

Une des conditions de la qualité de l'enseignement est la qualité des enseignants. D'où l'importance du développement professionnel des enseignants (DPE) et des actions, programmes et dispositifs destinés à influer sur ce développement. Aujourd'hui, les enseignants utilisent de manière généralisée les moyens numériques de communication et de collaboration, ce qui conduit un nombre croissant d'entre eux à participer à des réseaux connectés et des communautés en ligne. La question que nous posons ici est celle du rôle de ces réseaux connectés dans le DPE.

Nous rappellerons d'abord les composantes et les déterminants du DPE. Nous en viendrons ensuite aux réseaux connectés et finirons en montrant en quoi et dans quelles limites la participation à un réseau connecté contribue au développement professionnel.

1. Qu'est-ce que le développement professionnel des enseignants?

La notion de développement professionnel recouvre l'idée qu'au long de sa carrière, un professionnel change et apprend. Cependant, les chercheurs qui ont travaillé sur le DPE l'ont fait selon deux perspectives différentes.

La première perspective est descriptive. Elle met en évidence les étapes d'une évolution des individus, des phases successives de la «vie des enseignants » (Huberman, 1989). On s'y intéresse à « la modification des attitudes, des habiletés, des performances, des valeurs, de l'image de soi, du rapport au métier, de la perception à l'égard des élèves, des croyances, etc. » (Uwamariya \& Mukamurera, 2005, §10).

La seconde perspective met l'accent sur la professionnalisation, elle prend en compte l'interaction avec différents aspects du milieu professionnel et notamment la participation à des formations et elle insiste sur la construction et la maitrise des compétences nécessaires pour enseigner autant que sur les changements dans l'identité professionnelle. Enseigner est un métier qui s'apprend et la formation tout au long de la vie est un des déterminants du DPE. C'est dans cette seconde perspective que nous nous situons, celle des processus provoqués par la formation ou des dispositifs pouvant avoir une dimension de formation. Nous avons ainsi nous-même, en nous appuyant sur une série de travaux ${ }^{1}$, défini le

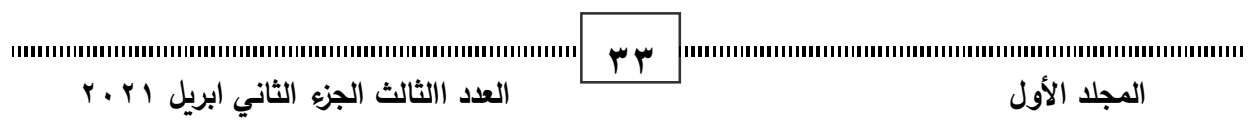


DPE « comme un processus individuel, structuré socialement, de changement de pratiques et d'acquisition progressive de compétences reconnues par la personne elle-même et par la communauté professionnelle dans laquelle la personne prend une part active et s'engage en vue d'améliorer l'apprentissage et la réussite scolaire des élèves » (Ferone \& Crinon, 2021).

Il existe plusieurs manières de peser délibérément sur le développement professionnel. Les plus classiques sont la transmission d'informations et de savoirs (lectures d'ouvrages ou d'articles, cours, conférences...), et la présentation d'exemples de manières de faire par des collègues, voire de modèles normatifs à imiter. Cela correspond au premier des trois processus de formation distingués par Gilles Ferry (1983/2003): un modèle de formation centré sur les acquisitions, par opposition à un modèle centré sur la démarche et à un autre centré sur l'analyse. Dans le modèle centré sur la démarche, il s'agit de se confronter à des expériences de vie, quelles qu'elles soient, qui mettent ses capacités à l'épreuve: "résoudre un problème, mettre en oeuvre un projet, aborder des situations imprévues, coopérer avec d'autres »... (Ferry, 1983/2003, p. 50). Dans le modèle centré sur l'analyse, la formation table sur «la capacité d'observer et d'observer les situations [...: études de cas, analyses d'expériences, observations de classes, de réunions pédagogiques, simulations, jeux de rôle, etc. » (Ferry, 1983/2003, p. 50).

L'accent mis dans le troisième modèle sur la capacité à analyser les situations pour faire face à l'imprévisibilité consubstantielle à l'exercice

${ }^{1}$ Day, 1999: Dionne, Lemyre \& Savoie-Zajc, 2010; Kaddouri, 2011; Lameul, Peltier \& Charlier, 2014; Leclerc \& Labelle, 2013; Marcel, 2005; Tardif, Lessard \& Lahaye, 1991; Uwamariya \& Mukamurera, 2005

de l'enseignement est à rapprocher des analyses du développement de la professionnalité proposées par plusieurs écoles de pensée.

La didactique professionnelle (Pastré, 2015; Pastré, Mayen \& Vergnaud, 2006) note ainsi le rôle du retour réflexif sur la pratique du métier et la compréhension des situations dans la constitution de l'expérience et l'adaptation des schemes organisateurs de l'activité à la

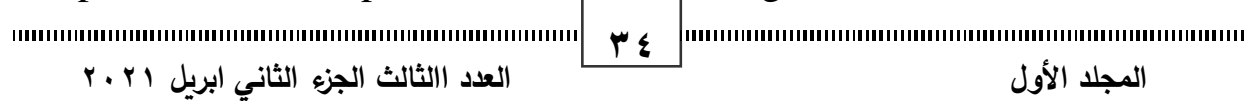


diversité des situations. «Il n'y a pas d'activité sans apprentissage. Mais [...] cela ne veut pas dire que l'activité productive et l'activité constructive possèdent le même empan temporel: l'activité productive s'arrête avec l'aboutissement de l'action, qu'il y ait réussite ou échec. L'activité constructive peut se continuer bien au-delà, quand notamment un sujet revient sur son action passée par un travail d'analyse réflexive pour la reconfigurer dans un effort de meilleure compréhension. » (Pastré, Mayen \& Vergnaud, 2006, § 42) Car l'expérience n'est pas le vécu et n'advient pas simplement par la récurrence de l'exercice d'une activité. Mais, dit de son côté Zeitler (2012) dans le prolongement de Dewey, sa construction dépend des capacités de l'acteur à interpréter son environnement.

De manière complémentaire, le courant de la clinique de l'activité met en évidence la dimension collective de l'activité professionnelle et le rôle du collectif dans la transmission du genre professionnel comme dans le développement des individus. Il insiste sur la mise en mots du travail, sur le dialogue qui permet de se mettre à distance de soi-même, de provoquer « un mouvement psychologique au cours duquel l'action vécue se métamorphose en opération permettant de vivre une autre action » (Clot et Faita, 2000, p. 34), qu'il s'agisse d'interagir avec des pairs au cours par exemple de « controverses professionnelles » (Clot, 2005) ou avec un formateur lors d'autoconfrontations, situations où l'opérateur réagit face à l'image de son activité filmée (Clot, 2014).

Le passage par l'écriture, dans le cas des enseignants, est un moyen de ce retour réflexif transformateur dont les différentes formes de mise en oeuvre ont été analysées par de multiples études (pour une revue, voir Crinon \& Guigue, 2006), depuis la mise en récit de sa pratique permettant de lui donner intelligibilité et sens (Cifali, 1996) jusqu'à la participation à des recherches collaboratives.

Une autre manière, assez différente, d'influer sur le développement professionnel des enseignants consiste à leur proposer des outils et supports de travail à utiliser avec leurs élèves, outils conçus en dialogue avec des praticiens à partir de savoirs issus de la recherche et accompagnés d'une explicitation de ces savoirs. L'appropriation de l'outil peut alors conduire à des processus de changement qui se traduisent par le transfert des techniques et des principes proposés dans l'outil

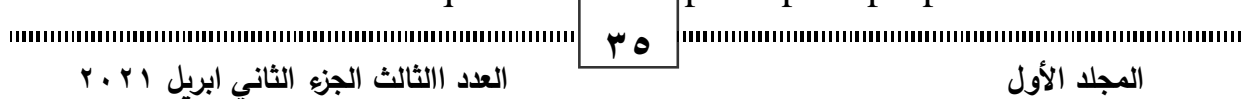


(Goigoux, Renaud \& Roux-Baron, à paraitre). On assiste à une genèse instrumentale décrite par la psychologie ergonomique (Béguin \& Rabardel, 2000): l'utilisation de l'outil transforme l'action des enseignants en même temps que ceux-ci le transforment.

Notons que les différentes approches actuelles, sans renoncer à la transmission, mettent en avant l'idée de collaboration. Celle-ci est au coeur aussi de la théorie de l'apprentissage situé et de la notion de communauté de pratique (Wenger, 1998), dont les membres construisent ensemble le sens de leurs pratiques professionnelles, créent de nouvelles pratiques et se constituent un « répertoire partagé ». Cette notion sera la référence de la plupart des communautés en ligne que nous allons étudier dans la suite de ce texte.

2. La participation à des réseaux connectés comme renouvèlement de la formation institutionnelle formelle et comme alternative à celle-ci.

L'accent mis sur les pratiques collaboratives est contemporain d'une mise en doute, au cours des années 90, de l'efficacité de la formation continue traditionnelle, donnée sous forme de sessions de formation au cours desquels des formateurs apportent des informations censées permettre aux participants de mettre à jour leurs connaissances sur une question. Ainsi la stratégie des formations négociées avec des équipes d'enseignants à partir de leurs demandes devait permettre « aux enseignants de renouveler et d'acquérir graduellement des savoirs dont ils ont besoin, ce qui constitue l'un des aspects de leur développement professionnel. Et, comme le soutiennent quelques auteurs (Clement \& Vandenberghe, 1999; Hargreaves \& Fullan, 1992; Perrenoud, 1994), cette acquisition n'est possible que lorsqu'il y a coopération, collaboration et culture collective au sein de l'établissement. » (Uwamariya \& Mukamurera, 2005, §38). Des études consacrées à des projets de formation collaborative ont montré leur impact sur les changements dans la pratique des enseignants qui y participaient (voir par exemple Butler, 2005).

C'est dans ce contexte que s'est considérablement développé, à partir des années 2000, le recours aux technologies numériques et à la formation à distance ou en partie à distance (formation hybride). C'est le cas d'actions de supervision pédagogique et de formations pilotées par

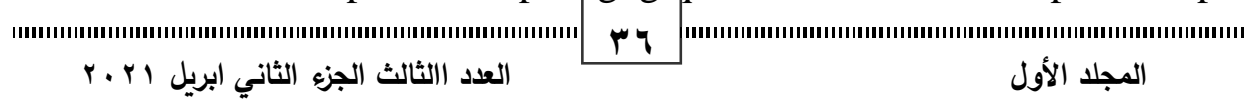


l'institution, telles celles étudiées par Villemontex et son équipe en France et dans des pays africains francophones (Nogry, Boulc'h \& Villemontex, 2019). C'est le cas aussi des communautés d'enseignants, formelles ou moins formelles qui ont fait l'objet d'études aujourd'hui nombreuses, que ce soit dans la sphère francophone (par exemple Baron \& Zablot, 2017: Daele, 2010; Quentin, 2014) ou dans la sphère anglophone (par exemple Bergviken Rensfeldt, Hillman \& Selwyn, 2018 ; Trust, Krutka \& Carpenter, 2016). Beaucoup de ces études se sont attachées à la question du développement professionnel (pour une revue, voir Lantz-Andersson, Lundin \& Selwyn, 2018).

L'utilisation du numérique a aussi donné lieu à de nombreuses initiatives spontanées nées du terrain et structurées par des associations. Ces réseaux revendiquent explicitement la portée de développement professionnel de leurs projets. C'est le cas de deux réseaux actifs dans la sphère francophone et que nous avons-nous-même étudiés: Twictée et Inversons la classe (Crinon \& Ferone, soumis; Ferone \& Crinon, 2020; Ferone \& Crinon, 2021). Le premier propose ainsi à ses membres « de se former de manière collaborative, sans injonction, sans préconisation, par le biais de la curiosité partagée ${ }^{2} \gg$ et l'autre donne pour visée aux échanges entre enseignants « inverseurs » une «(co)formation par les pairs $^{3} \gg$.

La question à laquelle nous voulons à présent tenter de répondre concerne cette ambition des réseaux connectés d'enseignants de contribuer au développement professionnel de leurs membres. Dans quelle mesure recouvre-t-elle une réalité?

3. L'hypothèse d'une contribution de la participation aux réseaux connectés au développement professionnel des enseignants est-elle vérifiée?

Nous nous appuierons principalement sur les analyses que nous avons consacrées au réseau Twictée et aux classes inversées. Le réseau Twictée rassemble des enseignants de l'école primaire et de l'école moyenne ${ }^{4}$ autour d'un projet d'amélioration de l'enseignement de l'orthographe. Il permet de faire vivre un dispositif très structuré de communication entre classes en utilisant Twitter: les élèves s'envoient par tweets des dictées et les corrections argumentées de ces dictées,

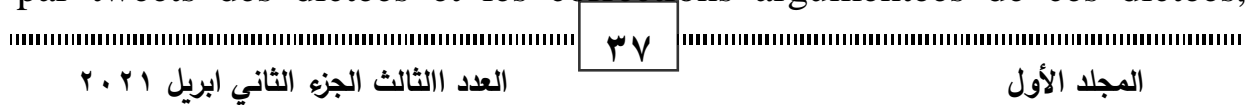


élaborées selon un modèle strict, afin de développer leur raisonnement orthographique. Les enseignants préparent les séances et les textes des dictées collectivement, à distance, sur un document partagé. Certains d'entre eux entretiennent aussi des échanges en utilisant d'autres outils de communication à distance mis à leur disposition par l'association (slack, twitter, messagerie) ou lors de rencontres en présence réunions de l'association, « cafés twictée », université d'été).

La classe inversée est une innovation qui touche l'ensemble des domaines disciplinaires enseignés et des niveaux d'enseignement, du primaire à l'enseignement supérieur. Il s'agit, dans sa version initiale nord-américaine, de modifier les frontières entre le travail des élèves en classe et hors de la classe: l'enseignant produit des « capsules » vidéo présentant les savoirs, que les élèves visionnent avant le cours, le temps en classe étant consacré aux exercices et aux interventions collaboratives. Nous avons cependant constaté, en étudiant les déclinaisons francophones de la classe inversée, une grande diversité dans la reprise du modèle. Les enseignants qui se revendiquent de la classe inversée, eux aussi, communiquent et collaborent entre eux, en partageant leurs capsules sur des plateformes, en participant aux activités proposées par l'association Inversons la classe, en échangeant sur le tweetchat Classe inversée ou encore de manière locale au sein d'un établissement scolaire.

Les études que nous avons produites se fondent sur l'analyse d'entretiens semi-directifs avec des enseignants pratiquant la Twictée (19

\footnotetext{
${ }^{2}$ https://www.twictee.org/

${ }^{3} \mathrm{http}: / / \mathrm{www}$.laclasseinversee.com/clic-classe-inversee-le-congres/

${ }^{4}$ C'est-à-dire, en France, le collège.
}

Enseignants trois fois à un an d'intervalle), des responsables de l'association Twictée (21 enseignants de l'école primaire ou de l'école moyenne), des enseignants inverseurs (11 enseignants français ou suisses de l'école moyenne, enseignant des disciplines diverses).

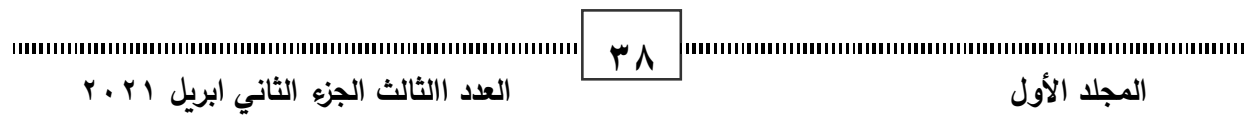


Que nous apprennent ces entretiens?

1. Ils mettent en évidence d'importants changements identitaires chez une partie des enseignants concernés. A l'origine de leur engagement dans le réseau, on observe un désir de trouver d'autres façons de travailler, de ne pas se cantonner à des pratiques routinières, d'éviter l'ennui. Ces enseignants ressentent le besoin de motiver leurs élèves en leur proposant des manières nouvelles de travailler, et parallèlement de retrouver eux-mêmes de l'intérêt pour le métier, intérêt qui s'était émoussé. Bref, participer à des réseaux innovants, disent-ils, leur a permis de renouveler leur gout d'enseigner.

Ce sont aussi des enseignants qui aiment travailler avec des collègues, à rebours de l'exercice solitaire du métier légué par la tradition (Lortie, 1975). Participer à un collectif, dans leur école ou en ligne, est un moyen de se sentir moins isolés. Et le réseau connecté est particulièrement apprécié par ceux qui affirment ne pas pouvoir échanger avec leurs collègues localement. On y trouve tout de suite des réponses aux questions qu'on se pose et qu'on y pose aux autres, affirment-ils. Et, au-delà de l'échange d'informations, c'est le lieu d'un support affectif. On se sent membre d'un groupe avec lequel on fait corps, surtout lorsqu'on se trouve en conflit avec d'autres collègues dont on ne partage pas la conception de l'enseignement.

De plus, la participation au réseau, pour ses membres les plus actifs, conduit à un élargissement des compétences, au-delà de la compétence à enseigner: découverte et maitrise de logiciels, animation du groupe d'adultes, organisation et gestion du travail associatif... Elle amène à présenter sa pratique à d'autres, lors de différentes réunions, d'universités d'été, de formations institutionnelles, à acquérir une reconnaissance, de la part de collègues, des parents, de la hiérarchie et à consolider ainsi son estime de soi. Elle constitue pour certains une étape dans une évolution professionnelle vers d'autres fonctions (formateur, directeur, inspecteur...).

2. La participation au réseau contribue aussi à changer les pratiques dans la classe, dans la mesure où il s'agit me mettre en oeuvre un dispositif nouveau : la twictée dans notre premier exemple, la classe inversée dans le second. On y prépare en commun un support (le texte de la dictée dans Twictée) ou bien on mutualise les supports des participants (les capsules vidéo des classes inversées). Dans ces dispositifs l'accent est mis sur le travail en petits groupes des élèves et la parole de ceux-ci, qui

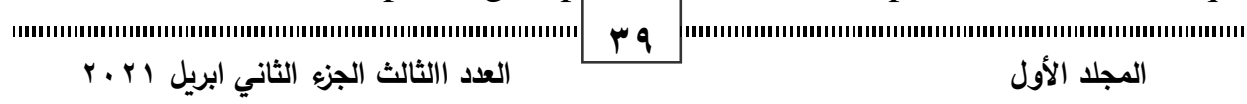


constituent aujourd'hui des doxas partagées par les enseignants (Crinon \& Ferone, 2018). Il ne semble d'ailleurs pas que les échanges entre enseignants permettent d'interroger ces doxas, ni l'efficacité du travail. Les entretiens indiquent qu'adopter ces pratiques pédagogiques correspond à une logique qui dépasse la transmission des contenus : ainsi dans le cas de Twictée, " elles contribuent à des objectifs transversaux, en particulier apprendre à coopérer, échanger, travailler avec d'autres ; elles procedent d'un type de pédagogie qui met l'accent sur la différenciation ou l'individualisation, à travers par exemple des "plans de travail" individualisés ou des feed-back évaluatifs » (Crinon, Ferone \& Font, 2021).

3. En revanche, la participation à ces réseaux semble produire peu d'apprentissages nouveaux chez les enseignants en ce qui concerne la mise à jour des contenus enseignés et la didactique des disciplines, c'està-dire la façon de transmettre ces contenus. Est-ce notamment pour cette raison que leurs élèves ne semblent pas mieux apprendre que ceux d'autres enseignants étrangers à ces réseaux innovants? En effet, dans le cas de Twictée, une évaluation des progrès des élèves dans les classes dont nous avons interviewé les enseignants ne montre pas de différences significatives avec les progrès réalisés dans des classes témoins (Brissaud, Viriot-Goeldel \& Ponton, 2019).

Les enseignants, quant à eux, se montrent pour beaucoup peu sensibles aux résultats des recherches récentes en didactique de la discipline concernée. Et lorsqu'ils évoquent les nouvelles connaissances qu'ils ont pu acquérir, ils font référence à des lectures ou des conférences, non à des échanges au sein du réseau, même si certains ont découvert des références de livres ou de postcasts dans le réseau, grâce à la veille documentaire effectuée par certains de ses membres.

Enfin, si ces réseaux, nous l'avons vu, sont le lieu de l'invention de nouveaux dispositifs et de la création de supports pédagogiques, on n'y trouve pas de discussions permettant de réfléchir sur les situations de classe après-coup, d'analyser le vécu, de revenir sur les difficultés rencontrées, d'avoir ces « controverses professionnelles » dont nous avons plus haut souligné l'apport au développement de la professionnalité. Ces constats rejoignent des résultats obtenus par d'autres chercheurs, "sur les difficultés des enseignants à engager en ligne des discussions approfondies sur leurs stratégies d'enseignement et

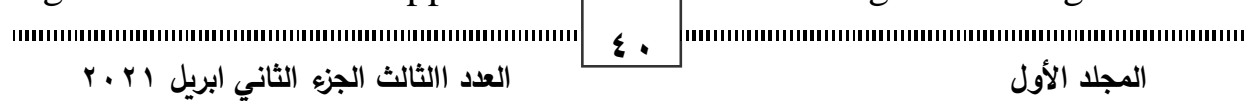


sur les apprentissages de leurs élèves (Macià \& García, 2016: Wells \& Feun, 2007), à confronter leurs idées et à s'opposer, notamment aux acteurs les plus en vue de la communauté (Brass \& Mecoli, 2011)» (Crinon \& Ferone, soumis).

4. Une quatrième leçon importante tirée de ces recherches est que les remarques précédentes ne valent pas de la même façon pour tous les participants aux réseaux. Car on constate des différences importantes entre les enseignants que nous avons interrogés.

- La familiarité avec les technologies et les outils numériques est un premier facteur, et surtout le désir d'en maitriser davantage les usages. S'engager dans un réseau connecté va de pair avec le souhait d'adopter en classe des pratiques qui font davantage appel au numérique. L'équipement de son établissement scolaire ou les injonctions officielles sont cités par certains comme des éléments déclencheurs de leur engagement. Mais le rapport au numérique joue aussi, à l'inverse, lorsqu'on abandonne le réseau: dans notre enquête sur la Twictée, qui s'est déroulée sur trois ans, plus de la moitié de notre échantillon avait mis fin à sa participation au cours de la période. Parmi les raisons données, la lourdeur du dispositif, tant de préparation commune en ligne que d'échanges entre élèves des classes partenaires par Twitter, revient fréquemment. L'intensité et la persévérance dans la participation et donc les effets qui s'en suivent ont à voir avec une pratique fluide des outils numériques.

- Le type d'animation du réseau est également en cause. Plusieurs des enseignants interrogés y insistent, les uns pour souligner le rôle facilitateur des créateurs de la Twictée dans l'animation de la communauté - ils savent créer un climat de confiance et de respect mutuel dans les échanges, d'autres pour regretter le caractère uniquement horizontal des échanges, qui ne permet pas de profiter des savoirs d'un formateur, ni de la prise de distance que son intervention permettrait.

- Le degré d'engagement des participants est un autre facteur qui conduit à des effets différenciés sur le développement professionnel. Chez certains, l'engagement est minimal, ils se définissent eux-mêmes parfois comme consommateurs - consommer des capsules produites par d'autres pour les inverseurs, se borner à inscrire sa classe dans le

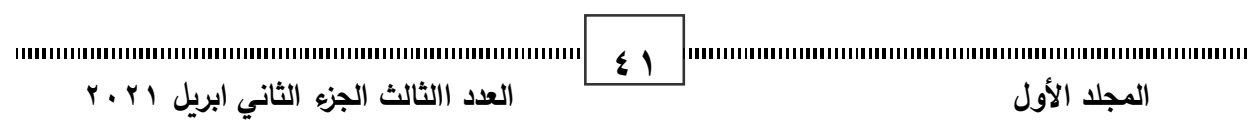


projet pour les «twictonautes». Ils sont peu présents dans les échanges au sein du réseau, parce qu'ils ne se sentent pas autorisés à y intervenir ou bien parce qu'ils gardent une distance critique. On retrouve là des phénomènes souvent mentionnés déjà dans la littérature. Ces « consommateurs » pourraient correspondre aux «lurkers » décrits par Preece, Nonnecke et Andrews (2004). L'attitude de retrait des « nouveaux membres » qui ne sentent pas légitimes a été également relevée (voir par exemple Alexander, Van Wyk, Bereng \& November, 2010).

D'autres participants au contraire, jouent un rôle de leadeurs dans l'organisation du réseau et l'animation des projets, postent des capsules, mettent à disposition des ressources, exercent une influence importante sur le groupe (voir Vangrieken, Meredith, Packer \& Kyndt, 2017). En fait, dans le cas de Twictée, « deux collectifs fonctionnent en parallèle ; le premier, les twictonautes, regroupe les enseignants qui interagissent avec leur classe pour effectuer les différents épisodes des twictées, le second regroupe les membres de l'association, qui participent ou non aux twictées, qui font fonctionner le dispos nombreux autres sujets : l'association propose ainsi plus d'une trentaine de canaux de discussion (slack) relatifs à des sujets propres à Twictée ou à des sujets plus généraux sur la pédagogie, le développement de carrière... » (Ferone \& Crinon, 2021). Les effets sur le développement professionnel, pour ces leadeurs, sont d'une bien plus grande ampleur.

- Il convient enfin d'insister sur la multiplicité des engagements de beaucoup des enseignants participants à des réseaux. Engagements parallèles ou successifs dans des projets qui parfois s'interpénètrent parce qu'on y retrouve les mêmes personnes: on va de l'un à l'autre, dans une recherche de nouveautés. Certains abandonnent un dispositif dès qu'ils ont l'impression qu'il se fige, quand d'autres y trouvent un intérêt renouvelé parce qu'ils y ont matière à de nouveaux investissements, à des prises de responsabilités, à des échanges plus intenses, à d'autres rencontres. D'autres aussi quittent un réseau lorsqu'ils pensent en avoir tiré tout ce qu'il y avait à en tirer. C'est ce qu'explicitent certains des enseignants interrogés, qui ont adapté à leur manière le dispositif Twictée, en fonction des besoins de leurs élèves. Cette capacité à modifier et adapter l'outil partagé d'abord au sein du

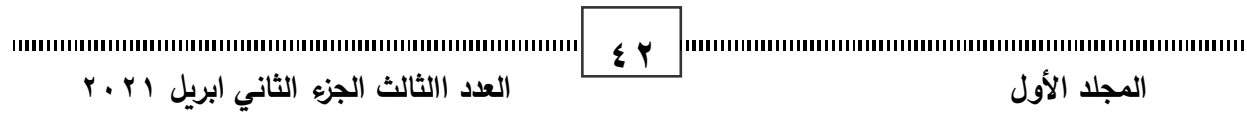


réseau peut d'ailleurs être considérée comme un signe d'acquisition de compétences.

Il est donc difficile d'évaluer la portée de la participation à un réseau connecté indépendamment des autres engagements d'un enseignant. Cette participation se combine à bien d'autres éléments, au sein d'interactions multiformes, pour contribuer à un trajet professionnel, comme l'ont déjà remarqué Liu, Miller et Jahng (2016). L'effet de la participation à un réseau, qu'il s'agisse d'effet direct ou d'un rôle déclencheur de formation, varie donc selon le moment où il intervient dans ce trajet propre à une personne.

\section{Conclusion}

Nous posions au début de ce texte la question de l'apport de la participation aux réseaux connectés « horizontaux » au développement professionnel des enseignants. Cet apport est réel, en particulier en termes de remaniement des identités. Mais il ne faudrait pas tomber dans l'illusion qu'il suffit de faire pour apprendre ni de participer à des projets au sein de réseaux pour se développer professionnellement. Le DPE implique aussi cette construction de l'expérience que permettent des échanges portant, au-delà des questions d'organisation, de préparation de la classe et d'ingénierie, sur les enjeux des situations et les problèmes qui se posent, au regard de ce qu'on peut entendre par amélioration de la qualité de l'enseignement: un enseignement qui permet à tous les élèves

${ }^{5}$ Loin de l'autopromotion et du dénigrement par des «trolls » constatés par Quentin (2019) dans son analyse de comptes Twitter d'enseignants. de progresser dans leurs apprentissages, y compris aux plus désavantagés par leur rapport, socialement construit, aux savoirs scolaires et aux modes de scolarisation. Participer à des réseaux connectés fournit donc des occasions de développement professionnel lorsqu'on y observe certaines activités: se référer aux savoirs issus de la recherche, expliciter ses buts, condition de l'autoregulation de ses apprentissages (Butler, 2005), travailler à l'analyse des situations et des cas d'élèves, s'appuyer sur le fonctionnement confiant de la communauté pour avoir des débats

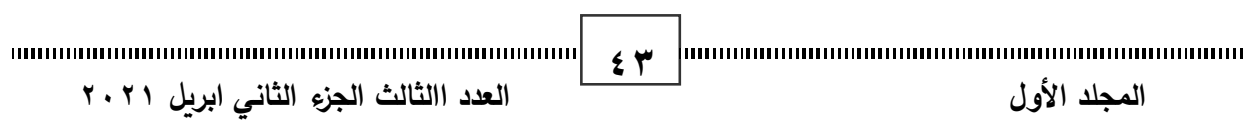


المجلة الدولية للتعليم الإكتروني

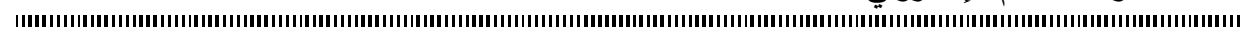
professionnels et faire de ses collègues des « amis critiques » (Day, 2002). 


\section{Références}

Alexander, G., Van Wyk, M., Bereng, T. \& November, I. (2010). Legitimate peripheral participation (LPP): The case for recognition of prior learning sites and knowledge in South Africa's transforming education system. Teaching and Teacher Education, 26(1), 45-52.

Baron, G.-L. \& Zablot, S. (2017). De la constitution de ressources personnelles à la création de communautés formelles: étude de cas en France. Review of science, mathematics and ICT education, University of Patras, 11(2), 27-45. hal-01671526

Béguin, P. \& Rabardel, P. (2000). Designing for instrument-mediated activity. Scandinavian Journal of Information Systems, 12(1), 173190. Bergviken Rensfeldt, A., Hillman, T. \& Selwyn, N. (2018). Teachers 'liking' their work? Exploring the realities of teacher Facebook groups. British Educational Research Journal, 44(2), 230-250.

Brass, J. \& Mecoli, S. (2011). The (failed) case of the Winston society wikispace: Challenges and opportunities of web 2.0 and teacher education. Contemporary Issues in Technology and Teacher Education, 11(2), 149-166.

Brissaud, C., Viriot-Goeldel, C. \& Ponton, C. (2019). Enseigner et apprendre l'orthographe avec la «Twictée ». Premiers résultats de l'évaluation d'un dispositif innovant d'enseignement de l'orthographe. Repères, 60, 107-130.

Butler, D. L. (2005). L'autoregulation de l'apprentissage et la collaboration dans le développement professionnel des enseignants. Revue des sciences de l'éducation, 31(1), 55-78. https://doi.org/10.7202/012358ar.

Cifali, M. (1996). Démarche clinique, formation et écriture. In L. Paquay, M. Altet, É. Charlier \& P. Perrenoud (Eds.), Former de enseignants professionnels (p. 119-135). Bruxelles: De Boeck.

Clement, M. \& Vandenberghe, R. (1999). Teachers' professional development: A solitary or collegial (ad)venture? Teaching and Teacher Education, 16, 81-101.

Clot, Y. (2005). L'auto-confrontation croisée en analyse du travail: l'apport de la théorie bakhtinienne du dialogue. Dans L. Filliettaz \& J.-P. Bronckart (dir.), L'analyse des actions et des discours en situation de travail (p. 37-55). Louvain-la-Neuve: Peeters.

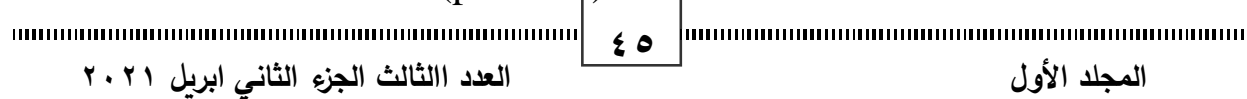


Clot, Y. (2014). Travail et pouvoir d'agir. Paris: Presses universitaires de France.

Clot, Y. \& Fasta, D. (2000). Genres et styles en analyse du travail: concepts et méthodes. Travailler, 4(7), 7-42.

Crinon, J. \& Ferone, G. (2018). Savoirs et conceptions professionnelles des enseignants, Education \& Formation, e-310, 39-57. [En ligne]: http://revueeducationformation.be

Crinon, J. \& Ferone, G. (soumis). Réseaux connectés d'enseignants et développement professionnel. Recherches en éducation.

Crinon, J., Ferone, G. \& Font, H. (2021, à paraitre). Les enseignants et l'orthographe, une enquête au cycle 3. Glottopol, 35. En ligne: http://glottopol.univ-rouen.fr/index.html

Crinon, J. \& Guigue, M. (2006). Écriture et professionnalisation. Revue française de Pédagogie, 156, 117-169.

Daele, A. (2010). Conditions et vécu du conflit sociocognitif au sein d'une communauté virtuelle d'enseignants: proposition d'un cadre d'analyse et étude de cas. Éducation et Formation, e-293, 65-80. En ligne:

http://revueeducationformation.be/index.php?revue $=9 \&$ page $=3$

Day, C. (2002). Developing teachers: The challenges of lifelong learning. London: Routledge

Falmer. Dionne, L., Lemyre, F. \& Savoie-Zajc, L. (2010). Vers une définition englobante de la communauté d'apprentissage (CA) comme dispositif de développement professionnel. Revue des sciences de l'éducation, 36(1), 25-43.

Ferone, G. \& Crinon, J. (2020). Interagir à distance dans une communauté d'enseignants: effets sur les conceptions relatives au numérique et à l'enseignement de l'orthographe. Recherches en éducation, $40, \quad 112-123 . \quad$ En ligne: https://journals.openedition.org/ree/460 Ferone, G. \& Crinon, J. (2021, à paraitre). Se former à distance de manière collaborative. Le cas du réseau Twictée. Médiations et médiatisations, 5. En ligne: http://revuemediations.teluq.ca

Ferry, G. (1983/2003). Le trajet de la formation. Paris: L'Harmattan.

Goigoux, R., Renaud, J. \& Roux-Baron, I. (à paraitre). Comment influencer positivement les pratiques pédagogiques de professeurs exérimenter? In B. Galand \& M. Janosz (Eds.), Verrous et leviers à l'amélioration des pratiques en éducation. Qu'en dit la recherche? 
Louvain-la-Neuve: Presses universitaires de Louvain.

Hargreaves, A. \& Fullan, M.G. (1992). Understanding teacher development. New York, NY: Teachers College Press.

Huberman, M. (1989). La vie des enseignants. Évolution et bilan d'une profession. Neuchâtel Paris: Delachaux et Niestlé.

Kaddouri, M. (2011). Motifs identitaires des formes d'engagement en formation. Savoirs, 25(1), 69-86.

Lameul, G., Peltier, C. \& Charlier, B. (2014). Dispositifs hybrides de formation et développement professionnel: effets perçus par des enseignants du supérieur. Education \& Formation, e-301, 99-113. [En ligne]: http://revueeducationformation.be

Lantz-Andersson, A., Lundin, M. \& Selwyn, N. (2018). Twenty years of online teacher communities: A systematic review of formallyorganized and informally-developed professional learning groups. Teaching and Teacher Education, 75, 302-315.

Leclerc, M. \& Labelle, J. (2013). Au coeur de la réussite scolaire: communauté d'apprentissage professionnelle et autres types de communautés. Education et francophonie, 41(2), 1-9. Liu, K., Miller, R. \& Jahng, K. E. (2016). Participatory media for teacher professional development: Toward a self-sustainable and democratic community of practice. Educational Review, 68(4), 420-443.

Lortie, D. C. (1975/2002). Schoolteacher: A sociological study. Chicago: The University of Chicago Press.

Macià, M. \& García, I. (2018). Professional development of teachers acting as bridges in online social networks. Research in Learning Technology, 26. DOI:10.25304/rt.v26.2057

Marcel, J.-F. (2005). Apprendre en travaillant. Contribution à une approche sociocognitive du développement professionnel de l'enseignant. Université de Toulouse II - Le Mirail.

Nogry, S., Boulc'h, L. \& Villemontex, F. (Dir.) (2019). Le numérique à l'école primaire. Pratiques de classe et supervision pédagogique dans les pays francophones. Villeneuve d'Asq: Presses universitaires du Septentrion.

Pastré, P. (2015). La didactique professionnelle approche anthropologique du développement chez les adultes. Paris: Presses universitaires de France.

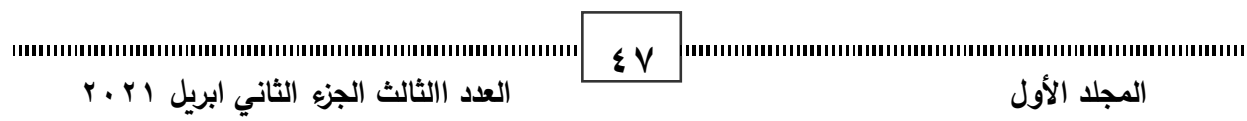


Pastré, P., Mayen, P. et Vergnaud, G. (2006). La didactique professionnelle. Revue française de pédagogie, 154, 145-198.

Perrenoud, P. (1994). La formation des enseignants, entre théorie et pratique. Paris: L'Harmattan.

Preece, J., Nonnecke, B. \& Andrews D. (2004). The top five reasons for lurking: improving community experiences for everyone. Computers in Human Behavior, 20(2), 201-223.

Quentin, I. (2014). Fonctionnements et trajectoires des réseaux professionnels en ligne: le cas des réseaux d'enseignants. Distance et médiations des savoirs, _ 7 [En ligne] :https://journals.openedition.org/dms/777

Quentin, I. (2019). Pratiques de communication d'enseignants sur Twitter et collectifs. Dans A. Beaune et al. (dir.), Collectifs en réseau d'enseignants producteurs de ressources. Rapport scientifique des laboratoires STEF et EDA dans le cadre de la convention DNE. [Rapport de recherche] Université Paris 5 Sorbonne Descartes; ENS Cachan. hal-02022830.

Tardif, M., Lessard, C. et Lahaye, L. (1991). Les enseignants des ordres d'enseignement primaire et secondaire face aux savoirs. Esquisse d'une problématique du savoir enseignant. Sociologie et société, 23(1), 55-69.

Trust, T., Krutka, D. G. \& Carpenter, J. P. (2016). Together we are better: Professional learning networks for teachers. Computers \& Education, 102, 15-34.

Uwamariya, A. \& Mukamurera, J. (2005). Le concept de « développement professionnel » en enseignement: approches théoriques. Revue des sciences de l'éducation, 31(1), 133-155. https://doi.org/10.7202/012361ar)

Vangrieken, K., Meredith, C., Packer, T. \& Kyndt, E. (2017). Teacher communities as a context for professional development: A systematic review. Teaching and Teacher Education, 61, 47-59.

Wells, C. \& Feun, L. (2007). Implementation of learning community principles: A study of six high schools. NASSP Bulletin, 91, 141160.

Wenger, E. (1998). Communities of practice: Learning, meaning, and identity. Cambridge, UK: Cambridge University Press.

Zeitler, A. (2012). Apprentissages interprétatifs et construction de l'expérience. Recherche et formation, 70, 31-46.

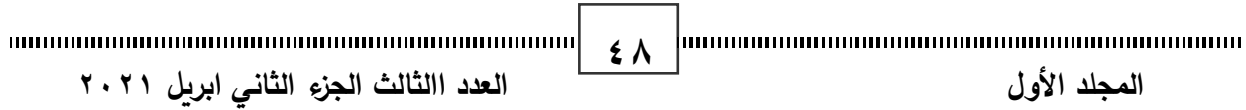

